$19,13,10$

\title{
Исследование влияния адгезии и ангармонизма колебаний атомов на тепловую проводимость границ „металл-диэлектрик“
}

\author{
() А.Г. Слепнёв \\ Московский государственный технический университет им. Н.Э. Баумана, \\ Москва, Россия \\ E-mail: andslepnev@yandex.ru
}

(Поступила в Редакцию 27 октября 2016 г.)

Представлен метод расчета тепловой проводимости границ „металл-диэлектрик“, учитывающий в рамках модели акустического несоответствия адгезионное взаимодействие и ангармонизм колебаний атомов на границе и позволяющий получить лучшее согласие расчетных данных с экспериментом.

DOI: 10.21883/FTT.2017.07.44612.403

\section{1. Введение}

Внедрение наноструктур в технике обусловливает актуальность проблемы теплопереноса через границы. Несмотря на значительное число выполненных работ $[1,2]$, в настоящее время не существует методов оценки коэффициента фононной тепловой проводимости границ, обеспечивающих приемлемое согласие расчета с экспериментом в широком диапазоне температур. Недостаточность учета лишь акустических [3] и диффузионных [4] несоответствий на границе очевидна. Так, эксперименты $[2,5,6]$ указывают на связь теплопереноса с адгезией. Учет же адгезии в расчетах [2,7] сопряжен с проблемой выбора потенциалов взаимодействия на границе. В представленной работе граничный теплоперенос рассмотрен в рамках модели акустического несоответствия с учетом адгезионного взаимодействия, параметры которого определялись в приближении модели $[8,9]$.

Открытым остается и вопрос об аномальной тепловой проводимости границ материалов с существенно различными акустическими свойствами $\left(\mathrm{Pb}-\mathrm{Al}_{2} \mathrm{O}_{3}, \mathrm{~Pb}\right.$-алмаз и др.). Данные эксперимента здесь на порядок выше теоретических оценок, что связывают с вкладом в теплоперенос либо ангармонизма [10-12], либо электронов металла $[13,14]$. В настоящей работе дан анализ влияния адгезии на существование локализованных упругих волн на границе и их вклада в теплоперенос через ангармоническое взаимодействие с объемными волнами.

Как отмечено выше, в настоящей работе рассмотрены границы между металлами и средами, не обладающими свободными электронами (далее именуемыми диэлектриками). Все переменные, относящиеся к металлам, обозначены индексом $j=1$, к диэлектрикам - индек$\operatorname{com} j=2$.

\section{2. Учет адгезионного взаимодействия в модели акустического несоответствия}

Коэффициент фононной тепловой проводимости через границу сред 1 и 2 в модели акустического несоответ- ствия [1,3] представляется в виде

$$
\begin{aligned}
\lambda_{S}= & \frac{k}{3} \sum_{e=1}^{3} \int_{0}^{\min \left(\omega_{D e 1}, \omega_{D e 2}\right) / c_{e 1}} \int_{0}^{\sqrt{\left(\omega / c_{e 1}\right)^{2}-\beta_{e 1}^{2}}} \theta_{e}\left(\left(\frac{\hbar \omega}{2 k T}\right)^{2}\right. \\
& \left.\times \sinh ^{-2}\left(\frac{\hbar \omega}{2 k T}\right)\right) c_{e 1} \cos \left(\chi_{e 1}\right) \frac{\pi d \xi^{2} d \beta_{e 1}}{(2 \pi)^{3}}
\end{aligned}
$$

где $e-$ поляризация падающей на границу упругой волны; $\omega-$ ее частота; $\xi$ и $\beta_{e j}-$ проекции волнового вектора на плоскость границы и нормаль к ней; $\chi_{e j}$ угол падения $\left(\cos \chi_{e j}=c_{e j} \beta_{e j} / \omega\right) ; c_{e j}-$ скорость звука; $k$ и $\hbar-$ постоянные Больцмана и Планка; $T$ температура;

$$
\omega_{D e j}=c_{e j} \sqrt[3]{6 \pi^{2} \rho_{j} / m_{j}}
$$

- частота Дебая; $\rho_{j}-$ плотность; $m_{j}-$ атомная масса. Коэффициент прохождения волны через границу сред с адгезионным взаимодействием [7,15] (без учета изменения поляризации волн на границе) имеет вид:

$$
\begin{aligned}
\theta_{e} & =\frac{\rho_{2} c_{e 2} \cos \left(\chi_{e 2}\right)}{\rho_{1} c_{e 1} \cos \left(\chi_{e 1}\right)} \frac{A_{e 2}}{A_{e 1}}\left(\frac{A_{e 2}}{A_{e 1}}\right)^{*} \\
& =4\left(\frac{\left(\mu_{e 1} \beta_{e 1}+\mu_{e 2} \beta_{e 2}\right)^{2}}{\mu_{e 2} \beta_{e 2} \mu_{e 1} \beta_{e 1}}+\frac{\mu_{e 2} \beta_{e 2} \mu_{e 1} \beta_{e 1}}{\kappa^{2}}\right)^{-1},
\end{aligned}
$$

где $A_{e j}-$ амплитуда волны $\left(A_{e j}^{*}-\right.$ комплексносопряженная амплитуда); $\mu_{e j} \approx \rho_{j} c_{e j}^{2}-$ модуль упругости в объеме; $\kappa$ - модуль упругости на границе.

Модуль упругости на границе $\kappa=\left(d^{2} W / d L^{2}\right)_{L=0}$ определен в рамках модели $[8,9]$, по которой взаимодействие сред 1 и 2 обусловлено их взаимной поляризацией, вызванной осцилляциями зарядов, а энергия взаимодействия определяется изменением энергии электромаг- 
нитного поля в зазоре между средами при изменении величины зазора $L$

$$
W(L)=\left\{\int_{0}^{\xi_{\max }} \frac{\hbar \Omega(\zeta, L)}{2} \frac{2 \pi \zeta d \zeta}{(2 \pi)^{2}}-\sum_{j=1}^{2} \int_{0}^{\xi_{\max } j} \frac{\hbar \Omega_{j}(\zeta)}{2} \frac{2 \pi \xi d \zeta}{(2 \pi)^{2}}\right\}
$$

где $\xi-$ проекция волнового вектора электромагнитной волны на плоскость границы, $\Omega_{j}(\xi)$ и $\Omega(\xi, L)-$ частота электромагнитных колебаний над свободной поверхностью $j$-той среды и в зазоре между средами соответственно.

Дисперсии $\Omega_{j}(\xi)$ и $\Omega(\xi, L)$ определяются из задачи о собственных значениях электромагнитных колебаний в зазоре и для границ металл $\left(\varepsilon_{1}=1-\left(\Omega_{p 1} / \Omega\right)^{2}\right)-$ диэлектрик $\left(\varepsilon_{2}=\right.$ const $\left.[16]\right)$ имеют вид [15]

$$
\Omega=\frac{\Omega_{p 1}}{\sqrt{2}} \sqrt{1-\frac{\left(\varepsilon_{2}-1\right)}{\left(\varepsilon_{2}+1\right)} \exp (-2 \xi L)},
$$

где $\Omega_{p 1}=\sqrt{\left(n_{e 1} q_{e}^{2} \rho_{1}\right) /\left(\varepsilon_{0} m_{e} m_{1}\right)}$ - частота плазменных колебаний в металле, $m_{e}$ и $q_{e}$ - масса и заряд электрона, $n_{e 1}$ - металлическая валентность, $m_{1}$ - масса атомов металла, $\varepsilon_{j}$ - диэлектрическая проницаемость, $\varepsilon_{0}$ - электрическая постоянная. Подстановка (4) в (3) приводит к выражению

$W(L)=\int_{\xi_{\min }}^{\xi_{\max }} \frac{\hbar}{2} \frac{\Omega_{p 1}}{\sqrt{2}}\left[\sqrt{1-\frac{\left(\varepsilon_{2}-1\right)}{\left(\varepsilon_{2}+1\right)} \exp (-2 \xi L)}-1\right] \frac{2 \pi \zeta d \xi}{(2 \pi)^{2}}$,

где $\xi_{\min }=\Omega_{p 1} /\left(C \sqrt{\varepsilon_{2}+1}\right) \quad-\quad$ определяется областью существования дисперсии (4), а $\xi_{\max }=$ $=\Omega_{p 1} /\left(v_{F 1} \sqrt{\varepsilon_{2}+1}\right) \quad$ - областью устойчивости плазменных колебаний в металле [9]; $C$ - скорость света в вакууме, $v_{F 1}=\left(\hbar / m_{e}\right) \sqrt[3]{3 \pi^{2} n_{e} \rho_{1} / m_{1}}-$ скорость электронов в металле на сферической поверхности Ферми. Поскольку $\xi_{\min } / \xi_{\max }=v_{F 1} / C \approx 10^{-2} \rightarrow 0$, нижний предел в (5) может быть положен равным нулю.

Данные для расчета взяты из источников $[4,17,18$ 21], результаты представлены в табл. 1-3. Полученные значения энергий адгезии $W_{0}=W(0)$, модулей упругости на границе $\kappa$ и коэффициентов тепловой проводимости $\lambda_{S}\left(\lambda_{S \kappa}-\right.$ приближение адгезионной и $\lambda_{S \infty}-$ приближение жесткой связи на границе) даны в табл. 1 и 3. Также в таблицах представлены экспериментальные данные $\lambda_{S \text { exp. }}$

В верхних строках табл. 1 приведены границы с

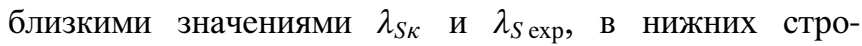

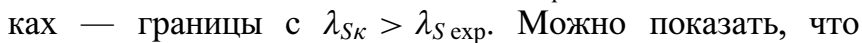
$\lambda_{S} \propto \theta_{e} \propto \kappa^{2} \propto W_{0}^{2}$ (при $\left.\kappa \rightarrow 0\right), \lambda_{S} \propto \theta_{e} \propto \kappa \propto W_{0}$ (при промежуточных $\kappa$ ) и $\lambda_{S} \propto \theta_{e} \propto A-B / W_{0}^{2}$ (при $\left.\kappa \rightarrow \infty\right)$, где $A$ и $B$ не зависят от $W_{0}$; и связать расхождение теории $\lambda_{S \kappa}$ с экспериментом $\lambda_{S \text { exp }}$, приведенное в нижних строках табл. 1, с завышенными в ряде случаев значениями $W_{0}$, которые важно сравнить с верхней оценкой
Таблица 1. Тепловая проводимость ряда границ при темпера-

\begin{tabular}{|c|c|c|c|c|}
\hline \multirow{2}{*}{ Граница } & \multirow{2}{*}{$W_{0}, \mathrm{~J} / \mathrm{m}^{2}$} & \multirow{2}{*}{$\kappa, \mathrm{Pa} / \mathrm{m}$} & \multicolumn{2}{|c|}{$\lambda_{S}, \mathrm{~W} /\left(\mathrm{K} \cdot \mathrm{m}^{2}\right)$} \\
\hline & & & $\lambda_{S} \kappa$ & $\lambda_{S \exp }$ \\
\hline $\mathrm{Al}-\mathrm{A} 1 \mathrm{~N}$ & 3.9 & $6.1 \cdot 10^{20}$ & $2.6 \cdot 10^{8}$ & $2.3 \cdot 10^{8}[22]$ \\
\hline $\mathrm{Al}-\mathrm{Al}_{2} \mathrm{O}_{3}$ & 3.2 & $4 \cdot 10^{20}$ & $1.9 \cdot 10^{8}$ & $2 \cdot 10^{8}[22,23]$ \\
\hline $\mathrm{A} 1-\mathrm{BaF}_{2}$ & 2.4 & $2.1 \cdot 10^{20}$ & $10^{8}$ & $10^{8}[23]$ \\
\hline $\mathrm{Al}-$ алмаз & 4.2 & $6.6 \cdot 10^{20}$ & $9.6 \cdot 10^{7}$ & $\begin{array}{l}4.6 \cdot 10^{7}[23] ; \\
1.8 \cdot 10^{8}[24]\end{array}$ \\
\hline $\mathrm{Au}-\mathrm{BaF}_{2}$ & 0.9 & $5.7 \cdot 10^{19}$ & $3.5 \cdot 10^{7}$ & $4 \cdot 10^{7}[23]$ \\
\hline $\mathrm{Ti}-\mathrm{Al}_{2} \mathrm{O}_{3}$ & 3.9 & $5.2 \cdot 10^{20}$ & $1.9 \cdot 10^{8}$ & $2 \cdot 10^{8}[23]$ \\
\hline Ti-алмаз & 5.0 & $8.6 \cdot 10^{20}$ & $10^{8}$ & $10^{8}[23]$ \\
\hline $\mathrm{Al}-\mathrm{GaN}$ & 3.8 & $5.6 \cdot 10^{20}$ & $5.2 \cdot 10^{8}$ & $1.9 \cdot 10^{8}[22]$ \\
\hline $\mathrm{Al}-\mathrm{Si}$ & 4.7 & $7.2 \cdot 10^{20}$ & $4.5 \cdot 10^{8}$ & $1.2 \cdot 10^{8}[22]$ \\
\hline $\mathrm{Cr}-\mathrm{AlN}$ & 9.2 & $2 \cdot 10^{21}$ & $6.9 \cdot 10^{8}$ & $2 \cdot 10^{8}[22]$ \\
\hline $\mathrm{Cr}-\mathrm{Al}_{2} \mathrm{O}_{3}$ & 7.5 & $1.3 \cdot 10^{21}$ & $5.9 \cdot 10^{8}$ & $1.9 \cdot 10^{8}[22]$ \\
\hline $\mathrm{Cr}-\mathrm{GaN}$ & 8.8 & $1.8 \cdot 10^{21}$ & $1 \cdot 10^{9}$ & $2.3 \cdot 10^{8}[22]$ \\
\hline $\mathrm{Cr}-\mathrm{Si}$ & 10.9 & $2.3 \cdot 10^{21}$ & $8.8 \cdot 10^{8}$ & $2 \cdot 10^{8}[22]$ \\
\hline
\end{tabular}
туре $300 \mathrm{~K}$

энергии адгезии [25]

$$
W_{a 0}=\sigma_{1}+\sigma_{2},
$$

где $\sigma_{1}, \sigma_{2}-$ поверхностные энергии металла и диэлектрика.

В табл. 2 приведены экспериментальные данные по $\sigma_{1}$ и $\sigma_{2}$, значения энергий адгезии $W_{0}(5)$ и $W_{a 0}(6)$ и соотношения $W_{0} / W_{a 0}$ и $\lambda_{S \kappa} / \lambda_{S \exp }$. В столбце $\lambda_{S \kappa} / \lambda_{S \exp }$ приведены также наиболее близкие пропорциональности $\lambda_{S} \propto W_{0}^{N}$ (для $N>1$ ) и $\lambda_{S} \propto A-B / W_{0}^{2}$. Исходя из таблицы, можно предположить, что

1) величина $W_{0}$ рассчитана верно, если $W_{0} / W_{a 0}=$ $=\lambda_{S \kappa} / \lambda_{S \exp } \approx 1$

2) величина $W_{0}$ завышена, если $W_{0} / W_{a 0} \approx \lambda_{S \kappa} / \lambda_{S \exp }>1$ и $\left(W_{0} / W_{a 0}\right)^{2} \approx \lambda_{S \kappa} / \lambda_{S \exp }>1$;

$3)$ если $W_{0} / W_{a 0}>\lambda_{S \kappa} / \lambda_{S \exp } \approx 1$, то величина $W_{0}$, возможно, завышена, что, однако, не влияет на равенство $\lambda_{S \kappa}$ и $\lambda_{S \exp }$, и соответствует случаю $\kappa \rightarrow \infty$ и $\lambda_{S} \approx A-B / W_{0}^{2}$.

Безусловно энергия адгезии $W_{a}=W_{a 0}-\sigma_{12}$ определяется также энергией границы $\sigma_{12}$, определяемой технологическим процессом. Так, в работе [25] энергии адгезии определены из экспериментов по смачиванию подложек жидкими металлами и имеют меньшие значения, чем величины $W_{0}$ и $W_{a 0}$, представленные в табл. 2 , что может говорить о неполной релаксации напряжений на границе или неполном покрытии каплей подложки. В работе [26], в зависимости от температуры отжига, энергия адгезии пленки $\mathrm{Au}$ к $\mathrm{SiO}_{2}$ менялась от $0.37 \mathrm{~J} / \mathrm{m}^{2}$ (после напыления) до $0.9 \mathrm{~J} / \mathrm{m}^{2}$ (отжиг при $100^{\circ} \mathrm{C}$ в течение $1 \mathrm{~h}$ ) и до $9.9 \mathrm{~J} / \mathrm{m}^{2}$ (отжиг при $300^{\circ} \mathrm{C}$ в течение $1 \mathrm{~h})$. И если отжиг при $100^{\circ} \mathrm{C}$ соответствовал снятию напряжений на границе, то отжиг при $300^{\circ} \mathrm{C}$ вызывал образование диффузионного интерфейса. Энергия же ад- 
Таблица 2. Поверхностные энергии (экспериментальные значения) и энергии адгезии (расчетные значения) для ряда веществ и их границ

\begin{tabular}{|c|c|c|c|c|c|c|}
\hline Граница & $\sigma_{1}, \mathrm{~J} / \mathrm{m}^{2}$ & $\sigma_{2}, \mathrm{~J} / \mathrm{m}^{2}$ & $W_{a 0}, \mathrm{~J} / \mathrm{m}^{2}$ & $W_{0}, \mathrm{~J} / \mathrm{m}^{2}$ & $W_{0} / W_{a 0}$ & $\lambda_{S \kappa} / \lambda_{S \exp }$ \\
\hline $\mathrm{Al}-\mathrm{AlN}$ & \multirow{7}{*}{$0.87[25]$} & $2[28]$ & 2.87 & 3.9 & 1.34 & 1.13 \\
\hline \multirow{2}{*}{$\mathrm{Al}-\mathrm{Al}_{2} \mathrm{O}_{3}$} & & $2.6[29] \alpha-\mathrm{Al}_{2} \mathrm{O}_{3}$ & 3.47 & \multirow[t]{2}{*}{3.2} & 0.92 & \multirow[t]{2}{*}{0.95} \\
\hline & & $1.7[29] \gamma-\mathrm{Al}_{2} \mathrm{O}_{3}$ & 2.57 & & 1.25 & \\
\hline $\mathrm{Al}-\mathrm{BaF}$ & & $0.28[30]$ & 1.15 & 2.4 & 2.09 & $1 ; \lambda_{S} \propto A-B / W_{0}^{2}$ \\
\hline $\mathrm{Al}-\mathrm{GaN}$ & & $1.5[31]$ & 2.37 & 3.8 & 1.6 & $2.74 ; \lambda_{S} \propto W_{0}^{2.2}$ \\
\hline $\mathrm{Al}-\mathrm{Si}$ & & $1.5[32]$ & 2.37 & 4.7 & 1.98 & $3.75 ; \lambda_{S} \propto W_{0}^{1.9}$ \\
\hline $\mathrm{Al-алмаз}$ & & $5.6[32]$ & 6.47 & 4.2 & 0.65 & 0.85 \\
\hline $\mathrm{Au}-\mathrm{BaF}_{2}$ & $1.15[25]$ & $0.28[30]$ & 1.43 & 0.9 & 0.63 & 0.875 \\
\hline $\mathrm{Cr}-\mathrm{AlN}$ & \multirow[t]{5}{*}{$1.64[25]$} & $2.0[28]$ & 3.64 & 9.2 & 2.53 & $3.45 ; \lambda_{s} \propto W_{0}^{1.4}$ \\
\hline $\mathrm{Cr}-\mathrm{Al}_{2} \mathrm{O}_{3}$ & & $2.6[29] \alpha-\mathrm{Al}_{2} \mathrm{O}_{3}$ & 4.24 & \multirow[t]{2}{*}{7.5} & \multirow[t]{2}{*}{1.77} & \multirow[t]{2}{*}{$3.08 ; \lambda_{S} \propto W_{0}^{2}$} \\
\hline & & $1.7[29] \gamma-\mathrm{Al}_{2} \mathrm{O}_{3}$ & 3.34 & & & \\
\hline $\mathrm{Cr}-\mathrm{GaN}$ & & $1.5[31]$ & 3.14 & 8.8 & 2.8 & $4.35 ; \lambda_{S} \propto W_{0}^{1.4}$ \\
\hline $\mathrm{Cr}-\mathrm{Si}$ & & $1.5[32]$ & 3.14 & 10.9 & 3.47 & $4.42 ; \lambda_{S} \propto W_{0}^{1.2}$ \\
\hline \multirow[t]{2}{*}{$\mathrm{Ti}-\mathrm{Al}_{2} \mathrm{O}_{3}$} & \multirow[t]{3}{*}{$1.53[25]$} & $2.6[29] \alpha-\mathrm{Al}_{2} \mathrm{O}_{3}$ & 4.13 & \multirow[t]{2}{*}{3.9} & 0.95 & \multirow[t]{2}{*}{0.95} \\
\hline & & $1.7[29] \gamma-\mathrm{Al}_{2} \mathrm{O}_{3}$ & 3.23 & & 1.21 & \\
\hline Ті-алмаз & & $5.6[32]$ & 7.13 & 5.0 & 0.7 & 1 \\
\hline
\end{tabular}

Таблица 3. Тепловая проводимость ряда границ материалов с существенно различными акустическими свойствами при температуре $300 \mathrm{~K}$

\begin{tabular}{|c|c|c|c|c|c|}
\hline \multirow{2}{*}{ Граница } & \multirow{2}{*}{$W_{0}, \mathrm{~J} / \mathrm{m}^{2}$} & \multirow{2}{*}{$\kappa, \mathrm{Pa} / \mathrm{m}$} & \multicolumn{3}{|c|}{$\lambda_{s}, \mathrm{~W} /\left(\mathrm{K} \cdot \mathrm{m}^{2}\right)$} \\
\hline & & & $\lambda_{S \kappa}$ & $\lambda_{S \infty}$ & $\lambda_{S \exp }$ \\
\hline $\mathrm{Au}-\mathrm{Al}_{2} \mathrm{O}_{3}$ & 1.3 & $1.08 \cdot 10^{20}$ & $5.5 \cdot 10^{6}$ & $3.8 \cdot 10^{7}$ & $4.5 \cdot 10^{7}[22,23]$ \\
\hline $\mathrm{Au}-\mathrm{Si}$ & 1.8 & $1.9 \cdot 10^{20}$ & $1.5 \cdot 10^{7}$ & $4.1 \cdot 10^{7}$ & $7.1 \cdot 10^{7}[22]$ \\
\hline $\mathrm{Au}-\mathrm{SiO}_{2}$ & 1.0 & $6.8 \cdot 10^{19}$ & $1.5 \cdot 10^{7}$ & $8.1 \cdot 10^{7}$ & $3.6 \cdot 10^{7}[5]$ \\
\hline $\mathrm{Au}-\mathrm{TiO}_{2}$ & 1.9 & $8.5 \cdot 10^{19}$ & $3.9 \cdot 10^{6}$ & $4.5 \cdot 10^{7}$ & $4 \cdot 10^{7}[6]$ \\
\hline Аu-алмаз & 1.6 & $1.8 \cdot 10^{20}$ & $3.2 \cdot 10^{6}$ & $1.9 \cdot 10^{7}$ & $4 \cdot 10^{7}[23]$ \\
\hline Ві-алмаз & 3.4 & $4.9 \cdot 10^{20}$ & $0.3 \cdot 10^{7}$ & $0.3 \cdot 10^{7}$ & $0.9 \cdot 10^{7}[12]$ \\
\hline $\mathrm{Cu}-\mathrm{SiO}_{2}$ & 1.4 & $1 \cdot 10^{20}$ & $1 \cdot 10^{8}$ & $4.4 \cdot 10^{8}$ & $4.3 \cdot 10^{8} \quad[6]$ \\
\hline $\mathrm{Mo}-\mathrm{Al}_{2} \mathrm{O}_{3}$ & 6.0 & $9.6 \cdot 10^{20}$ & $2.5 \cdot 10^{8}$ & $4.1 \cdot 10^{8}$ & $6.7 \cdot 10^{8}[36]$ \\
\hline $\mathrm{Pb}-\mathrm{Al}_{2} \mathrm{O}_{3}$ & 2.5 & $2.8 \cdot 10^{20}$ & $4.7 \cdot 10^{6}$ & $7.2 \cdot 10^{6}$ & $5.5 \cdot 10^{7}[23]$ \\
\hline $\mathrm{Pb}-\mathrm{BaF}_{2}$ & 1.9 & $1.45 \cdot 10^{20}$ & $3.2 \cdot 10^{7}$ & $5.2 \cdot 10^{7}$ & $6.5 \cdot 10^{7}[23]$ \\
\hline $\mathrm{Pb}-$ алмаз & 3.2 & $4.6 \cdot 10^{20}$ & $2.3 \cdot 10^{6}$ & $3 \cdot 10^{6}$ & $1.8 \cdot 10^{7}[12] ; 3 \cdot 10^{7}[23]$ \\
\hline $\mathrm{Pt}-\mathrm{AlN}$ & 3.1 & $4.26 \cdot 10^{20}$ & $3.2 \cdot 10^{7}$ & $7 \cdot 10^{7}$ & $1.2 \cdot 10^{8}[10]$ \\
\hline $\mathrm{Pt}-\mathrm{Al}_{2} \mathrm{O}_{3}$ & 2.5 & $2.8 \cdot 10^{20}$ & $2.2 \cdot 10^{7}$ & $7.7 \cdot 10^{7}$ & $1.2 \cdot 10^{8}[10]$ \\
\hline $\mathrm{Pt}-\mathrm{Si}$ & 3.6 & $5 \cdot 10^{20}$ & $4.8 \cdot 10^{7}$ & $7.7 \cdot 10^{7}$ & $1.4 \cdot 10^{8}[22]$ \\
\hline
\end{tabular}

Пр имеч чнн ие. В табл. 3 представлены границы, для которых расчетные значения $\lambda_{S \kappa}$ оказываются меныше экспериментальных. В таблице также приведены расчетные значения $\lambda_{S \infty}$, полученные в приближении жесткой связи $(\kappa \rightarrow 0)$ на границе.

гезии металлов к керамике, определенная в работе [27], имеет значения, близкие к данным в табл. 2.

Поскольку $W_{0} \propto \Omega_{p 1} \xi_{\max }^{2} \propto \Omega_{p 1}^{3} / v_{F}^{2} \propto n_{e l}^{5 / 6}$, можно полагать, что завышенные значения $W_{0}$ связаны с использованием в расчетах максимальной валентности метал- лов [19]. Тем не менее, исследования $d$-металлов [33,34] указывают на более сложную зависимость $\varepsilon(\Omega)$, чем $\varepsilon=1-\left(\Omega_{p} / \Omega\right)^{2}$. Так, в [33] показано, что $\Omega_{p} \rightarrow \Omega_{p s}$ при $\Omega \rightarrow 0$, и $\Omega_{p} \rightarrow \Omega_{p d}$ при $\Omega \rightarrow \infty$, где $\Omega_{p s}$ и $\Omega_{p d}-$ частоты плазменных колебаний $s$ - и $d$-электронов. В [34] 


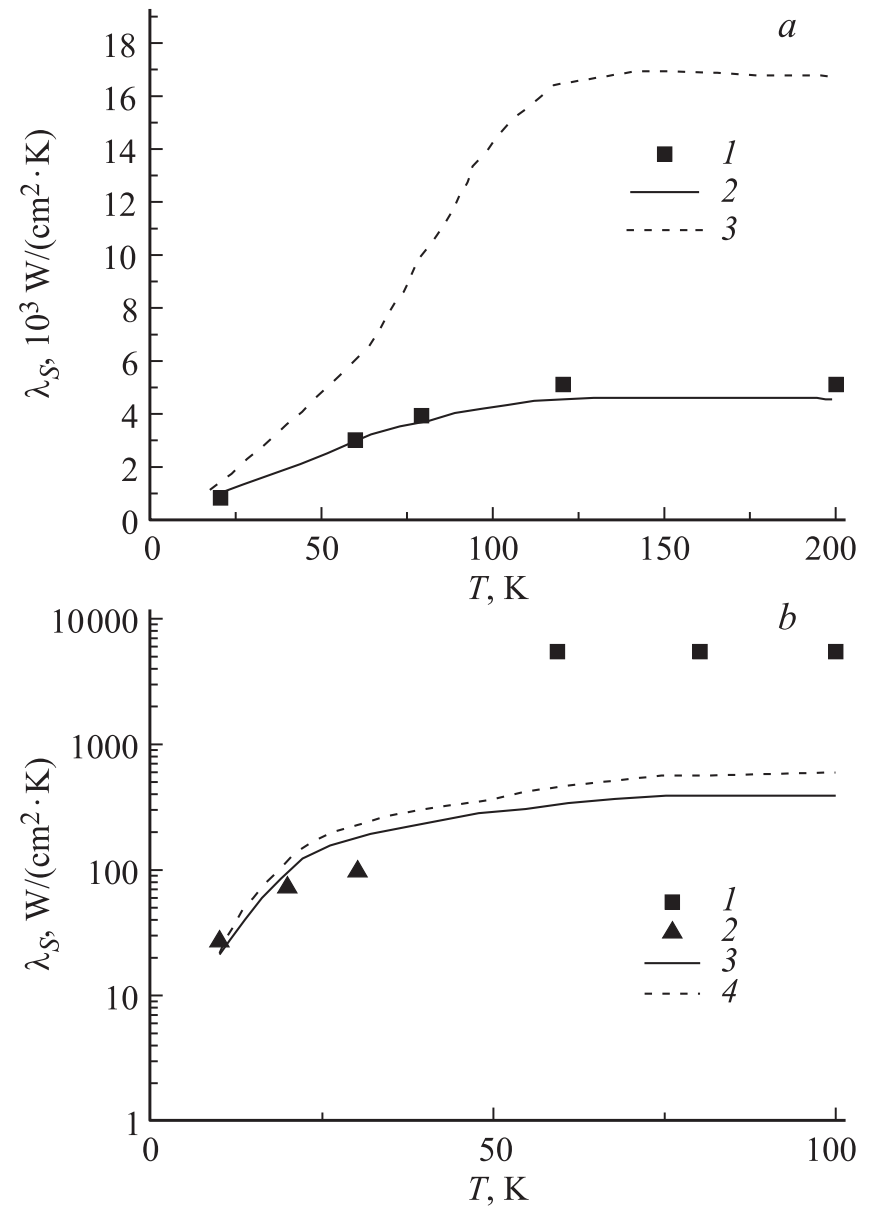

Рис. 1. Зависимость тепловой проводимости границ от температуры: $a-\mathrm{Au}-\mathrm{BaF}_{2}: 1-$ эксперимент [23], $2-$ расчет в приближении адгезионной связи, 3 - расчет в приближении жесткой связи $(\kappa \rightarrow \infty) ; b-\mathrm{Pb}-\mathrm{Al}_{2} \mathrm{O}_{3}$ ( $\lambda_{S}$ в логарифмическом масштабе): 1 - эксперимент [23], 2 - эксперимент [37], 3 - расчет в приближении адгезионной связи, $4-$ расчет в приближении жесткой связи $(\kappa \rightarrow \infty)$.

указаны два резонанса, соответствующих возбуждениям $s$ - и $d$-электронов. Вероятно, сильнее связанные с ядром $d$-электроны дают меньший вклад в $W_{0}$ по сравнению с $s$-электронами [35], что не учтено в данной работе.

На рис. 1, $a$ и $b$ приведено сравнение температурных зависимостей коэффициентов тепловой проводимости $\lambda_{S}$ границ $\mathrm{Au}-\mathrm{BaF}_{2}$ и $\mathrm{Pb}-\mathrm{Al}_{2} \mathrm{O}_{3}$, рассчитанных в приближении адгезионной $\lambda_{S \kappa}$ и жесткой $\lambda_{S \infty}$ связей, с экспериментальными данными $\lambda_{S}$ ехр. Из рисунка видно, что при высоких температурах в случае $\mathrm{Au}-\mathrm{BaF}_{2}$ (табл. 1) экспериментальные данные лучше описываются моделью, учитывающей адгезию на границе, в то же время в случае $\mathrm{Pb}-\mathrm{Al}_{2} \mathrm{O}_{3}$ (табл. 3) теоретические оценки лежат ниже экспериментальных данных.

Границы, приведенные в табл. 3, образованы материалами с существенно различными акустическими свойствами (например, $c_{t} \mathrm{~Pb}=700 \mathrm{~m} / \mathrm{s}$, a $c_{t \mathrm{Al}_{2} \mathrm{O}_{3}}=6350 \mathrm{~m} / \mathrm{s}$ ) и интересны тем, что сегодня нет общепринятого объ- яснения, почему их тепловая проводимость при высоких температурах выше даже идеальных теоретических оценок $[1,2]$. Так, из рис. $1, b$ видно, что расчет согласуется с экспериментом при малых температурах, но при температурах выше дебаевской $(\sim 100 \mathrm{~K}$ для свинца [23]) эксперимент на порядок превосходит теорию. Не останавливаясь на возможном влиянии электронов металла на теплоперенос через границу $[13,14]$, рассмотрим подробнее влияние на него взаимодействия упругих волн [10-12]. Действительно, простейшая дисперсия Лява [38] для двухслойной среды $\left(h_{j}-\right.$ толщина $j$-того слоя)

$$
\mu_{1} \beta_{1} \sin \left(h_{1} \beta_{1}\right) \cos \left(h_{2} \beta_{2}\right)+\mu_{2} \beta_{2} \sin \left(h_{2} \beta_{2}\right) \cos \left(h_{1} \beta_{1}\right)=0,
$$

$$
\beta_{j}=\sqrt{\left(\omega / c_{t j}\right)^{2}-\xi^{2}}
$$

описывает волны, существующие как во всем объеме системы $\beta_{2}^{2}>0 \quad\left(c_{t 1}<c_{t 2}\right)$, так и локализованные в слое с меньшей скоростью звука $\beta_{2}^{2}<0$. Увеличение соотношения $c_{t 2} / c_{t 1}$ ведет к расширению в фазовом пространстве области локализованных волн. Соотношение фазовых объемов, соответствующих локализованным и нелокализованным волнам, можно оценить как

$$
n \approx\left[\left(\omega / c_{t 1}\right)^{3}-\left(\omega / c_{t 2}\right)^{3}\right]\left(c_{t 2} / \omega\right)^{3} \approx\left(c_{t 2} / c_{t 1}\right)^{3},
$$

откуда $n_{\mathrm{Pb}_{-} \mathrm{Al}_{2} \mathrm{O}_{3}} \approx 750$ при $c_{t \mathrm{~Pb}}=700 \mathrm{~m} / \mathrm{s}$ и $c_{t \mathrm{Al}_{2} \mathrm{O}_{3}}=$ $=6350 \mathrm{~m} / \mathrm{s}$. Таким образом, можно ожидать значительного усиления теплопереноса через границу, если в системе существуют механизмы, способные перебрасывать волны из области локализации.

\section{3. Учет интерфейсных ангармонических явлений}

Из рис. $1, b$ видно, что тепловая проводимость границы $\mathrm{Pb}-\mathrm{Al}_{2} \mathrm{O}_{3}$ начинает проявлять свой аномальный характер при температурах, несколько меньших температуры Дебая свинца. Можно предположить, что из-за слабости адгезионной связи ангармонизм колебаний в свинце на интерфейсе проявляется раньше, чем в объеме. Если при этом существуют интерфейсные волны, то на них вероятны рассеяние и делокализация волн, локализованных в объеме свинца.

Получим дисперсию интерфейсных волн, полагая тело 2 абсолютно жестким $\left(c_{2} \gg c_{1}\right)$. Потенциалы растяжения-сжатия и сдвига имеют вид

$$
\begin{gathered}
\varphi_{1}=\Phi_{1} \exp (i(\xi x-\omega t)) \exp \left(-\alpha_{10} z\right), \\
\psi_{1}=\Psi_{1} \exp (i(\xi x-\omega t)) \exp \left(-\beta_{10} z\right), \quad \varphi_{2}=0, \quad \psi_{2}=0, \\
\text { где } \alpha_{10}=\sqrt{\xi^{2}-\left(\omega / c_{l 1}\right)^{2}}, \beta_{10}=\sqrt{\xi^{2}-\left(\omega / c_{t 1}\right)^{2}} .
\end{gathered}
$$

Граничные условия на интерфейсе $(z=0)$ представим следующим образом: $u_{1 z} \kappa=\sigma_{1 z z}$ и $u_{1 x} \kappa=\sigma_{1 z x}$, где 
$u_{1}$ - поле смещений, $\sigma_{z z 1}$ и $\sigma_{z x 1}-$ поля напряжений на границе, $\lambda_{1}$ и $\mu_{1}$ - коэффициенты Ламе [38]

$$
\begin{gathered}
\mathbf{u}_{1}=\operatorname{grad} \varphi_{1}+\operatorname{rot} \boldsymbol{\Psi}_{1}, \\
\sigma_{z z 1}=\lambda_{1}\left(\frac{\partial u_{x 1}}{\partial x}+\frac{\partial u_{z 1}}{\partial z}\right)+2 \mu_{1} \frac{\partial u_{z 1}}{\partial z}, \\
\sigma_{z x 1}=\mu_{1}\left(\frac{\partial u_{x 1}}{\partial z}+\frac{\partial u_{z 1}}{\partial x}\right) .
\end{gathered}
$$

Дисперсионное соотношение для интерфейсных волн на границе слабосвязанных упругого 1 и абсолютно жесткого 2 полупространств имеет вид

$$
\begin{aligned}
& \xi^{2}(\kappa\left.+2 \mu_{1} \beta_{10}\right)\left(\kappa+2 \mu_{1} \alpha_{10}\right)-\left(\alpha_{10} \kappa-\lambda_{1}\left(\frac{\omega}{c_{l 1}}\right)^{2}+2 \mu_{1} \alpha_{10}^{2}\right) \\
& \times\left(\beta_{10} \kappa+\mu_{1}\left(\beta_{10}^{2}+\xi^{2}\right)\right)=0
\end{aligned}
$$

которое переходит в дисперсию волн Рэлея [38] при $\kappa \rightarrow 0$ :

$$
4 \xi^{2} \beta_{10} \mu_{1} \alpha_{10}-\left(2 \mu_{1} \alpha_{10}^{2}-\lambda_{1}\left(\frac{\omega}{c_{l 1}}\right)^{2}\right)\left(\beta_{10}^{2}+\xi^{2}\right)=0 .
$$

Анализ дисперсии (7) показал, что интерфейсные волны вероятны на всех границах, приведенных в табл. 3, за исключением границ с поливалентными металлами $(\mathrm{Pb}, \mathrm{Bi}, \mathrm{Mo})$, валентность которых в расчетах (5) была взята максимальной, что могло привести к завышенным значениям коэффициента $\kappa$ и некорректным заключениям о возможности локализации волн на интерфейсе.

Проведем анализ рассеяния упругих волн на границе сред 1 и 2 с интерфейсным слоем толщины $\delta$, представляющим собой среду 1 с измененными вследствие ангармонизма модулями упругости. Пренебрежем изменением поляризации волн на границе. Граничные условия запишем в виде $z=0$ - граница среды 2 с интерфейсным слоем

$$
\left\{\begin{array}{l}
\left(\mu_{1}+\Delta \mu_{1}\right)\left(\frac{\partial u_{S}}{\partial z}\right)_{z=0}=\mu_{2}\left(\frac{\partial u_{2}}{\partial z}\right)_{z=0}, \\
\left(u_{S}-u_{2}\right)_{z=0} \kappa=\mu_{2}\left(\frac{\partial u_{2}}{\partial z}\right)_{z=0},
\end{array}\right.
$$

$z=\delta$ - граница среды 1 с интерфейсным слоем

$$
\left\{\begin{array}{l}
\left(\mu_{1}+\Delta \mu_{1}\right)\left(\frac{\partial u_{S}}{\partial z}\right)_{z=\delta}=\mu_{1}\left(\frac{\partial u_{1}}{\partial z}\right)_{z=\delta}, \\
\left(u_{S}\right)_{z=\delta}=\left(u_{1}\right)_{z=\delta},
\end{array}\right.
$$

где $\Delta \mu_{1}$ - возмущение объемного модуля упругости в интерфейсном слое, связанное с ангармонизмом интерфейсных волн, $u_{1}$ и $u_{2}$ - поля смещений атомов в объемах сред 1 и 2, $u_{S}$ - поле смещений атомов в интерфейсном слое, обусловленное падением волн из среды 1 на границу.
Полагая для упрощения амплитуду интерфейсных волн (7) неизменной в пределах слоя их затухания $\delta$, представим возмущение $\Delta \mu_{1}$ в виде

$$
\begin{aligned}
\Delta \mu_{1} & =M_{1} \frac{\partial U_{S}}{\partial x} \\
& =M_{1} \sum_{S} \xi_{S} A_{S}\left(\exp \left(i \xi_{S} x\right)+\exp \left(-i \xi_{S} x\right)\right) \exp \left(-i \omega_{S} t\right),
\end{aligned}
$$

где $M_{1}$ - некий модуль упругости 3-го порядка, $U_{S}-$ упрощенное представление поля смещений атомов в интерфейсной волне (7). При этом $U_{S} \neq u_{S}$, поскольку $u_{S}$ - поле смещений в интерфейсном слое, вызываемое волнами, падающими из объема. Поля смещений $u_{1}, u_{S}$, $u_{2}$ представим в виде [39]

$$
\begin{aligned}
& u_{1}=\left(A_{1} \exp \left(i \beta_{1} z\right)+B_{1} \exp \left(-i \beta_{1} z\right)\right) \exp (i(\xi x-\omega t)) \\
&+\sum_{J} B_{1 J} \exp \left(-i \beta_{1 J} z\right) \exp \left(i\left(\xi_{J} x-\omega_{J} t\right)\right)=u_{10}+\sum_{J} u_{1 J}, \\
& u_{2}= A_{2} \exp \left(i \beta_{2} z\right) \exp (i(\xi x-\omega t))+\sum_{J} A_{2 J} \exp \left(i \beta_{2 J} z\right) \\
& \quad \times \exp \left(i\left(\xi_{J} x-\omega_{J} t\right)\right)=u_{20}+\sum_{J} u_{2 J}, \\
& u_{S}=\left(A_{1} \exp \left(i \beta_{1} z\right)+B_{1} \exp \left(-i \beta_{1} z\right)\right) \exp (i(\xi x-\omega t)) \\
& \quad+\sum_{J}\left[A_{S J} \exp \left(i \beta_{S J} z\right)+B_{S J} \exp \left(-i \beta_{S J} z\right)\right] \\
& \quad \times \exp \left(i\left(\xi_{J} x-\omega_{J} t\right)\right)=u_{10}+\sum_{J} u_{S J} .
\end{aligned}
$$

Подставляя $u_{1}, u_{S}, u_{2}$ и $\Delta \mu_{1}$ в граничные условия, полагая возмущение $\Delta \mu_{1}$ малым, пренебрегая величинами большего порядка малости $\Delta \mu_{1} u_{S J}$ и приводя слагаемые с одинаковыми показателями экспонент, получим объединение систем уравнений

$$
\left[\begin{array}{l}
\left\{\begin{array}{l}
\mu_{1} \beta_{1}\left[A_{1}-B_{1}\right]=\mu_{2} \beta_{2} A_{2}, \\
\left(A_{1}+B_{1}-A_{2}\right) \kappa=\mu_{2} i \beta_{2} A_{2},
\end{array}\right. \\
\left\{\begin{array}{l}
\mu_{1} \beta_{S J}\left[A_{S J}-B_{S J}\right]+\xi_{S} M_{1} \beta_{1} A_{S}\left[A_{1}-B_{1}\right]=\mu_{2} \beta_{2 J} A_{2 J} \\
\left(A_{S J}+B_{S J}-A_{2 J}\right) \kappa=\mu_{2} i \beta_{2 J} A_{2 J} \\
\mu_{1} \beta_{S J}\left[A_{S J} \exp \left(i \beta_{S J} \delta\right)-B_{S J} \exp \left(-i \beta_{S J} \delta\right)\right] \\
\quad+M_{1} \beta_{1} \xi_{S} A_{S}\left[A_{1} \exp \left(i \beta_{1} \delta\right)-B_{1} \exp \left(-i \beta_{1} \delta\right)\right] \\
=-\mu_{1} \beta_{1 J} B_{1 J} \exp \left(-i \beta_{1 J} \delta\right) \\
A_{S J} \exp \left(i \beta_{S J} \delta\right)+B_{S J} \exp \left(-i \beta_{S J} \delta\right)=B_{1 J} \exp \left(-i \beta_{1 J} \delta\right)
\end{array}\right.
\end{array}\right.
$$

где первая система описывает линейное прохождение волн через невозмущенную границу, а последующие системы описывают рассеяние падающих на границу волн с генерацией волн, для которых выполняются условия $\xi_{J}=\xi \pm \xi_{S}, \omega_{J}=\omega+\omega_{S}$ и

$$
\beta_{j J}=\sqrt{\left(\left(\omega+\omega_{S}\right) / c_{j}\right)^{2}-\left(\xi \pm \xi_{S}\right)^{2}} \quad(j=1,2, S) .
$$

Из последней системы при условиях $\beta_{S J} \approx \beta_{1 J}\left(\Delta \mu_{1} \rightarrow 0\right)$ и $\delta \rightarrow 0$ получим связь между амплитудами волн па- 
дающей $A_{1}$ и сгенерированной вследствие взаимодействия $A_{2 J}$

$$
\frac{A_{2 J}}{A_{1}}=\frac{-2 i \frac{M_{1} \beta_{1}^{2} \xi_{S} A_{S} \delta}{\mu_{1} \beta_{1 J}}\left(\frac{\mu_{1} \beta_{1}}{\mu_{2} \beta_{2}}+i \frac{\mu_{1} \beta_{1}}{\kappa}-\frac{\beta_{1 J}}{\beta_{1}}\right)}{\left(\frac{\mu_{2} \beta_{2 J}}{\mu_{1} \beta_{1 J}}+1+i \frac{\mu_{2} \beta_{2 J}}{\kappa}\right)\left(\frac{\mu_{1} \beta_{1}}{\mu_{2} \beta_{2}}+1+i \frac{\mu_{1} \beta_{1}}{\kappa}\right)} .
$$

Определим тепловой поток в среде 2, учитывая генерацию волн на границе

$$
\begin{aligned}
q= & \frac{1}{3} \sum_{e \omega \xi} \frac{\rho_{2} \omega_{J}^{2}}{2}\left(\bar{u}_{2}+\sum u_{2 J}\right)\left(\bar{u}_{2}+\sum u_{2 J}\right)^{*} c_{2} \cos \left(\chi_{2 J}\right) \\
\approx & \frac{1}{3} \sum_{e \omega \xi} \frac{\rho_{2} \omega_{J}^{2}}{2}\left(\bar{A}_{2} \bar{A}_{2}^{*}+\bar{A}_{2}^{*} \sum A_{2 J}+\bar{A}_{2} \sum A_{2 J}^{*}\right) \\
& \times c_{2} \cos \left(\chi_{2 J}\right),
\end{aligned}
$$

где $\bar{A}_{2}-$ амплитуды волн $\bar{u}_{2}$, испытавших линейное прохождение через границу и обладающих характеристиками $\beta_{2 J}$ и $\omega_{J} ; A_{2 J}$ - амплитуды сгенерированных волн $u_{2 J}, \sum A_{2 J} \sum A_{2 J}^{*} \rightarrow 0$ как величина большего порядка малости. Суммы $\sum_{J}$ берутся по всем сгенерированным волнам с характеристиками $\beta_{2 J}$ и $\omega_{J}$.

Представим выражение для теплового потока в виде

$$
\begin{aligned}
q= & \frac{1}{3} \sum_{e \omega \xi} \frac{\rho_{2} \omega_{J}^{2}}{2} \bar{A}_{2} \bar{A}_{2}^{*}\left\{1+\sum\left[\left(\frac{A_{2 J}}{A_{1}}\right)\left(\frac{A_{1}}{\bar{A}_{1}}\right)\left(\frac{\bar{A}_{1}}{\bar{A}_{2}}\right)\right]\right. \\
& \left.+\sum\left[\left(\frac{A_{2 J}^{*}}{A_{1}^{*}}\right)\left(\frac{A_{1}^{*}}{\bar{A}_{1}^{*}}\right)\left(\frac{\bar{A}_{1}^{*}}{\bar{A}_{2}^{*}}\right)\right]\right\} c_{2} \cos \left(\chi_{2 J}\right),
\end{aligned}
$$

где

$$
\bar{A}_{2} / \bar{A}_{1}=2 /\left(\frac{\mu_{2} \beta_{2 J}}{\mu_{1} \beta_{1 J}}+1+\frac{\mu_{2} i \beta_{2 J}}{\kappa}\right)
$$

- определяются линейным прохождением волн $\bar{u}_{2}$ через границу, а $A_{2 J} / A_{1}$ определяется формулой (8).

Подставляя эти выражения в произведение $\left(A_{2 J} / A_{1}\right)\left(\bar{A}_{1} / \bar{A}_{2}\right)$ и учитывая, что волны $u_{2 J}$ и $\bar{u}_{2}$ обладают одним набором $\beta, \xi, \omega$, а также положив для упрощения $\beta_{2} \approx i \xi$ (поскольку интересен переброс локализованных волн при $\left.c_{1} \ll c_{2}\right)$, получим

$$
\begin{aligned}
& \left(\frac{A_{2 J}}{A_{1}}\right)\left(\frac{\bar{A}_{1}}{\bar{A}_{2}}\right)=\frac{M_{1} \beta_{1}^{2} \xi_{S} A_{S} \delta}{\mu_{1} \beta_{1 J}} \\
& \times \frac{\left(\frac{\mu_{1} \beta_{1}}{\kappa}-\frac{\mu_{1} \beta_{1}}{\xi \mu_{2}}\right)\left(1+\frac{\beta_{1 J}}{\beta_{1}}\right)+i \frac{\beta_{1 J}}{\beta_{1}}-i\left(\frac{\mu_{1} \beta_{1}}{\kappa}-\frac{\mu_{1} \beta_{1}}{\xi \mu_{2}}\right)^{2}}{\left(1+\left(\frac{\mu_{1} \beta_{1}}{\kappa}-\frac{\mu_{1} \beta_{1}}{\xi \mu_{2}}\right)^{2}\right)} .
\end{aligned}
$$

Приняв амплитуды $\bar{A}_{1}$ и $A_{1}$ действительными и положив их отношение выражением

$$
A_{1} / \bar{A}_{1}=\sqrt{\omega_{J}\left(\exp \left(\frac{\hbar \omega_{J}}{k T}\right)-1\right) / \omega\left(\exp \left(\frac{\hbar \omega}{k T}\right)-1\right)},
$$

перепишем (9) в виде

$$
q=\frac{1}{3} \sum_{e \omega \xi} \frac{\rho_{2} \omega_{J}^{2}}{2} \bar{A}_{2} \bar{A}_{2}^{*}\{1+Y\} c_{2} \cos \left(\chi_{2 J}\right),
$$

где $Y-$ соотношение вкладов волн $\bar{u}_{2}$ и $\sum u_{2 J}$ в тепловой поток (соотношение ангармонической и гармонической составляющих теплового потока)

$$
\begin{aligned}
Y= & 2 \sum \frac{M_{1} \beta_{1}^{2} \xi_{S} A_{S} \delta}{\mu_{1} \beta_{1 J}} \frac{\left(\frac{\mu_{1} \beta_{1}}{\kappa}-\frac{\mu_{1} \beta_{1}}{\xi \mu_{2}}\right)\left(1+\frac{\beta_{1 J}}{\beta_{1}}\right)}{\left(1+\left(\frac{\mu_{1} \beta_{1}}{\kappa}-\frac{\mu_{1} \beta_{1}}{\xi \mu_{2}}\right)^{2}\right)} \\
& \times \sqrt{\frac{\omega_{J}\left(\exp \left(\frac{\hbar \omega_{J}}{k T}\right)-1\right)}{\omega\left(\exp \left(\frac{\hbar \omega}{k T}\right)-1\right)}} .
\end{aligned}
$$

Перейдем в (10) к интегрированию. В интересующем нас случае $c_{1} \ll c_{2}$ и $c_{S} \approx c_{1}$ (следует из (7)) очевидно, что $\beta_{2 J}^{2}>0$ (условие прохождения сгенерированной волны $u_{2 J}$ через границу) выполнимо только при $\xi_{J}=\xi-\xi_{S}$, что дает возможность преобразовать

$$
\beta_{2 J}=\sqrt{\left(\left(\omega+\omega_{S}\right) / c_{2}\right)^{2}-\left(\xi \pm \xi_{S}\right)^{2}}
$$

к выражению

$$
\begin{aligned}
\xi & =\xi_{S}+\left(\frac{\omega+\omega_{S}}{c_{2}}\right) \sin \left(\chi_{2 J}\right) \\
& =\left(\frac{\omega_{J}-\omega}{c_{S}}\right)+\left(\frac{\omega_{J}}{c_{2}}\right) \sin \left(\chi_{2 J}\right),
\end{aligned}
$$

определяющему на дисперсионной диаграмме (рис. 2,a) точки, соответствующие падающим волнам с параметрами $\omega$ и $\xi$, генерирующим проходящие через границу волны с параметрами $\omega_{J}$ и $\chi_{J}$. Из (11) следует, что рассматриваемая падающая волна $u_{10}$ при взаимодействии с интерфейсной волной $U_{S}$ генерирует лишь одну проходящую волну $u_{2 J}$ (рис. $2, a$ ), в то время как интерфейсная волна $U_{S}$ участвует в генерации нескольких проходящих волн (рис. $2, b$ ), взаимодействуя с другими падающими волнами. Максимальная $\omega_{\max }$ (условие $\xi=0$ ) и минимальная $\omega_{\min }$ (условие $\xi=\omega_{\min } / c_{S}$ ) частоты падающих волн, участвующих в генерации проходящих через границу волн, определяются из (11) выражениями

$$
\begin{gathered}
\omega_{\max }= \begin{cases}\omega_{J}\left(1+\left(\frac{c_{S}}{c_{2}}\right) \sin \left(\chi_{2 J}\right)\right), & \omega_{\max }<\omega_{D 1}, \\
\omega_{D 1}, & \omega_{\max }>\omega_{D 1},\end{cases} \\
\omega_{\min }=\frac{\omega_{J}}{2}\left(1+\left(\frac{c_{S}}{c_{2}}\right) \sin \left(\chi_{2 J}\right)\right) .
\end{gathered}
$$

Таким образом, суммирование в (10) по всем сгенерированным волнам частоты $\omega_{J}$ заменим интегрированием на плоскости „, $\xi-\omega$ “ по частоте падающих волн $\omega$ при 

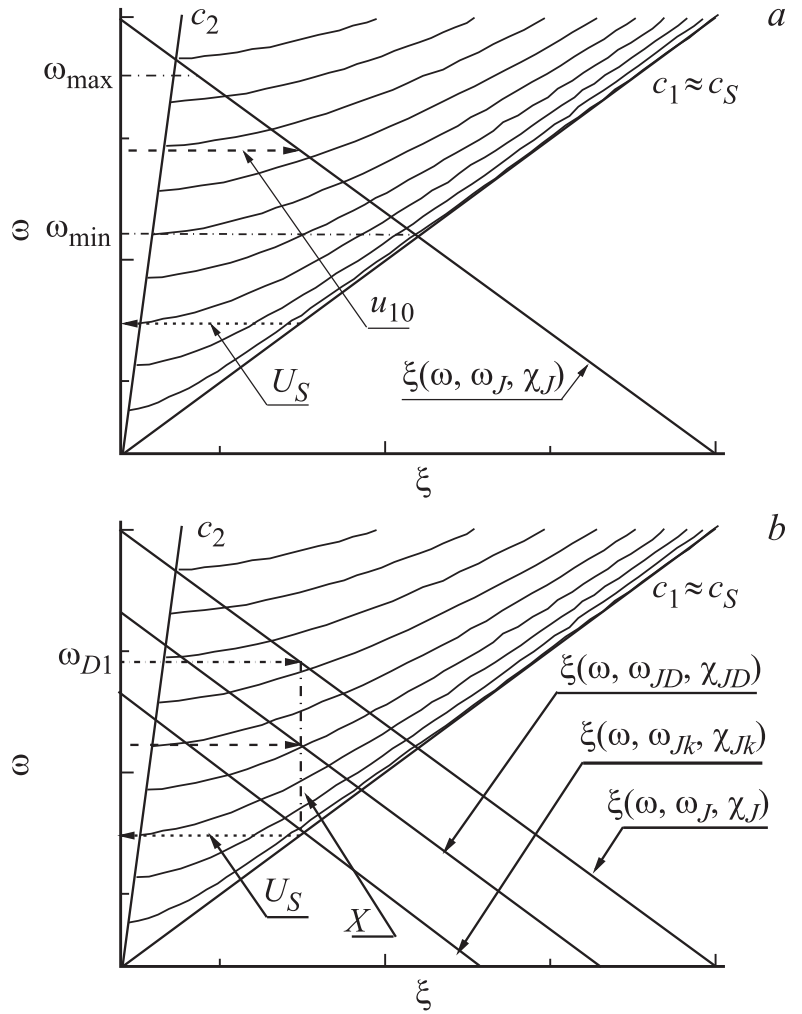

Pис. 2. a) Схема взаимодействия падающей $u_{10}$ и интерфейсной $U_{S}$ волн с генерацией проходящей волны $u_{2 j}$; $b)$ Схема взаимодействия интерфейсной волны $U_{S}$ с различными падающими волнами и генерации различных мод $u_{2 J}$. Отрезок $X \approx \omega_{D 1}-c_{S} \xi_{S}-$ геометрическое место проекций $\xi$ волновых векторов падающих волн, взаимодействие с которыми приводит к генерации проходящих волн $u_{2 j}$; $\omega / c_{2}<\xi<\omega / c_{1}-$ область волн, локализованных в среде 1 ; $0<\xi<\omega / c_{2}-$ область волн, проходящих через границу; $\xi\left(\omega, \omega_{J}, \xi_{J}\right)-$ прямая (11), $\omega_{D 1}$ - частота Дебая среды 1.

заданном значении $\omega_{J}$ в пределах $\left[\omega_{\min }, \omega_{\max }\right]$ вдоль прямой $\xi(\omega)$, определяемой выражением (11)

$$
\begin{aligned}
Y= & \frac{2 M_{1}}{\mu_{1}} \int_{\omega_{\min }}^{\omega_{\max }} \frac{\beta_{1}^{2} \delta \xi_{S} A_{S}\left(\frac{\mu_{1} \beta_{1}}{\kappa}-\frac{\mu_{1} \beta_{1}}{\xi \mu_{2}}\right)\left(1+\frac{\beta_{1 J}}{\beta_{1}}\right)}{\beta_{1 J}\left(1+\left(\frac{\mu_{1} \beta_{1}}{\kappa}-\frac{\mu_{1} \beta_{1}}{\xi \mu_{2}}\right)^{2}\right)} \\
& \times \sqrt{\frac{\omega_{J}\left(\exp \left(\frac{\hbar \omega_{J}}{k T}\right)-1\right)}{\omega\left(\exp \left(\frac{\hbar \omega}{k T}\right)-1\right)} \frac{d \omega}{\Delta \omega} \tau,}
\end{aligned}
$$

где $1 / \tau \approx X / \Delta \omega=\left(\omega_{D 1}-c_{S} \xi_{S}\right) / \Delta \omega-$ коэффициент, учитывающий ослабление интерфейсной волны $U_{S}$ вследствие генерации проходящих волн в диапазоне частот $X$ с интервалом $\Delta \omega$ (рис. $2, b)$. Существуют также процессы, приводящие к генерации волн, вновь локализующихся в среде 1, что означает отсутствие переноса энергии через границу сред и ее последующее перераспределение между колебательными модами среды 1 , поэтому эти процессы не учитываются в ослаблении падающих и интерфейсных волн в выражении (12).
Для оценки $Y$ дополнительно использовались $M_{1} \approx\left(2 \gamma_{1}+3\right) \mu_{1}$ - модуль упругости 3-го порядка [38], $\gamma_{1}$ - параметр Грюнайзена [40], $\delta \xi_{S} \approx 1$ (при условии $\left.c_{2} \gg c_{S} \approx c_{1}\right)$. Зависимость $Y$ от температуры для различных частот $\omega_{J}$ приведена на рис. 3.

Оценим отношение тепловой проводимости $\lambda$, полученной с учетом ангармонизма, к тепловой проводимости $\lambda_{0}$, полученной в приближении акустического несоответствия, выражением

$$
\frac{\lambda}{\lambda_{0}}=\frac{\sum_{e \xi \omega} \rho_{2} \omega_{J}^{2} \bar{A}_{2} \bar{A}_{2}^{*}\{1+Y\} c_{2} \cos \left(\chi_{2 J}\right)}{\sum_{e \xi \omega}^{\omega<\omega_{D 1}} \rho_{2} \omega_{J}^{2} \bar{A}_{2} \bar{A}_{2}^{*} c_{2} \cos \left(\chi_{2 J}\right)} .
$$

Соотношение $\lambda / \lambda_{0}$ для различных границ, как функция аргумента $\rho_{1} c_{1} \omega_{D 1} / \kappa=\rho_{1}\left(c_{1 L} \omega_{D 1 L}+2 c_{1 T} \omega_{D 1 T}\right) /(3 \kappa)$, характеризующего влияние адгезии на прохождение волн через границу (2), представлено на рис. 4.

Из рис. 4 видно, что представленная модель в ряде случаев позволила получить неплохое согласие теоретических оценок с экспериментальными данными, а также получить зависимость $\lambda / \lambda_{0}$ от $\rho_{1} c_{1} \omega_{D 1} / \kappa$, качественно близкую к зависимости $\lambda_{S} \exp / \lambda_{S \kappa}$, основанной на экспериментальных данных.
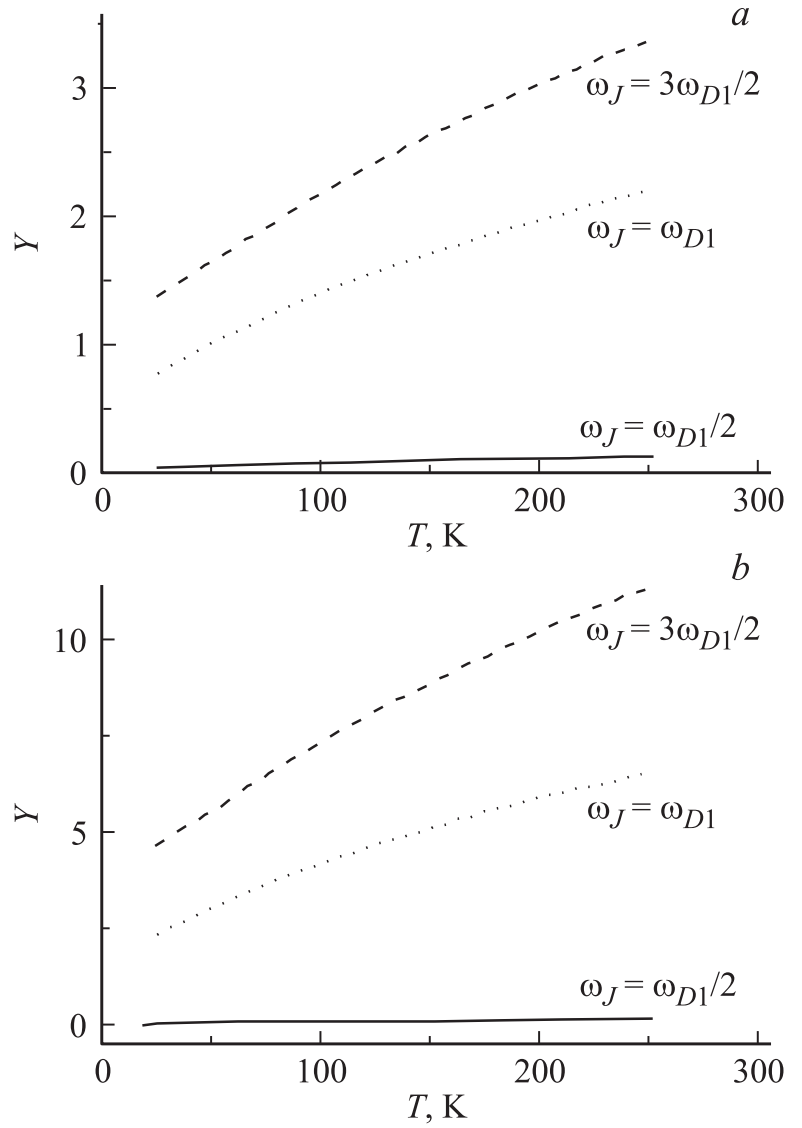

Рис. 3. Соотношение вкладов ангармонических и гармонических колебаний в тепловой поток через границу в зависимости от температуры на различных частотах в системе $\mathrm{Pb}-\mathrm{Al}_{2} \mathrm{O}_{3}$ ( $a$ - продольные волны, $b-$ поперечные волны). 


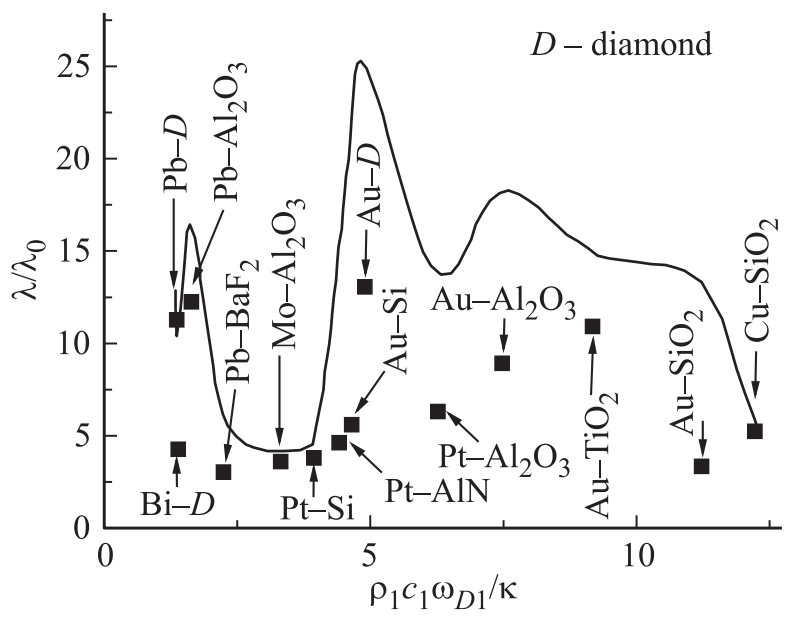

Рис. 4. Соотношение $\lambda / \lambda_{0}$ : сплошная кривая - расчет по формуле (13); точки - оценка $\lambda / \lambda_{0}=\lambda_{S \exp } / \lambda_{S \kappa}$ на основе данных, представленных в табл. 3.

В заключение анализа возможного влияния интерфейсного ангармонизма на теплоперенос через границы стоит отметить недавно вышедшую работу [41], в которой методами молекулярной динамики показано, что вклад интерфейсного ангармонизма в теплоперенос через границу $\mathrm{Si}-\mathrm{Ge}$ (вещества с достаточно близкими величинами скоростей звука) может составлять 15\%.

\section{4. Заключение}

В представленном исследовании установлено, что учет в расчетах адгезионного взаимодействия на границе позволяет улучшить согласие расчетных и экспериментальных значений тепловой проводимости границы. Показано, что важным эффектом при описании тепловой проводимости границ (в особенности материалов с существенно различными акустическими свойствами) может оказаться ангармонический переброс локализованных мод через границу. В заключение важно отметить, что рассмотренные два механизма, влияющие на теплоперенос через границу, являются лишь малой частью спектра явлений, происходящих на границе и в той или иной мере влияющих на теплоперенос. Кроме этого, модели, использованные в данной работе для описания адгезии и ангармонизма на границе, являются крайне упрощенными, и их следует рассматривать в большей степени как качественные, чем количественные.

\section{Список литературы}

[1] D.G. Cahill, W.K. Ford, K.E. Goodson, G.D. Mahan, A. Majumdar, H.J. Maris, R. Merlin, S.R. Phillpot. Appl. Phys. Rev. 93, 793 (2003).

[2] D.G. Cahill, P.V. Braun, G. Chen, D.R. Clarke, S. Fan, K.E. Goodson, P. Keblinski, W.P. King, G.D. Mahan, A. Majumdar, H.J. Maris, S.R. Phillpot, E. Pop, L. Shi. Appl. Phys. Rev. 1, 011305 (2014).

[3] И.М. Халатников. ЖЭТФ 22, 687 (1952).
[4] E.T. Swartz, R.O. Pohl. Rev. Mod. Phys. 61, 605 (1989).

[5] M.D. Losego, M.E. Grady, N.R. Sottos, D.G. Cahill, P.V. Braun. Nature. Mater. 11, 502 (2012).

[6] P.J. O’Brien, S. Shenogin, J. Liu, P.K. Chow, D. Laurencin, P.H. Mutin, M. Yamaguchi, P. Keblinski, G. Ramanath. Nature Mater. 12, 118 (2013).

[7] R. Prasher. Appl. Phys. Lett. 94, 041905 (2009).

[8] И.Е. Дзялошинский, Е.М. Лифшиц, Л.П. Питаевский. УФН 73, 381 (1961).

[9] А.Н. Вакилов, М.В. Мамонова, В.В. Прудников. ФТТ 39, 964 (1997).

[10] P.E. Hopkins, P.M. Norris, R.J. Stevens. J. Heat Trans. 130, 022401 (2008).

[11] Y.A. Kosevich. Phys. Rev. B 52, 1017 (1995).

[12] H.K. Lyeo, D.G. Cahill. Phys. Rev. B 73, 144301 (2006).

[13] A.V. Sergeev. Phys. Rev. B 58, R10199 (1998).

[14] M.L. Huberman, A.W. Overhauser. Phys. Rev. B 50, 2865 (1994).

[15] А.Г. Слепнев, В.И. Хвесюк. Наноинженерия 1, 17 (2011).

[16] G. Xu, M. Tazawa, P. Jin, S. Nakao, K. Yoshimura. Appl. Phys. Lett. 82, 3811 (2003).

[17] L. Bergström. Adv. Colloid Interface Sci. 70, 125 (1997).

[18] Физические величины. Справочник / Под ред. И.С. Григорьева, Е.3. Мейлихова. Энергоатомиздат, М. (1991). $1232 \mathrm{c}$.

[19] В.К. Григорович. Электронное строение и термодинамика сплавов железа. Наука, М. (1970). 292 с.

[20] C. Deger, E. Bom, H. Angerer, O. Ambacher, M. Stutzmann, J. Homsteiner, E. Riha, G. Fischherauer. Appl. Phys. Lett. 72, 2400 (1998).

[21] Y.N. Xu, W.Y. Ching. Phys. Rev. B 48, 4335 (1993).

[22] R.J. Stevens, A.N. Smith, P.M. Norris. J. Heat Trans. 127, 315 (2005).

[23] R.J. Stoner, H.J. Maris. Phys. Rev. B 48, 16373 (1993).

[24] M. Battabyal, O. Beffort, S. Kleiner, S. Vaucher, L. Rohr. Diamond Rel. Mater. 17, 1438 (2008).

[25] В. Миссол. Поверхностная энергия раздела фаз в металлах. Металлургия, М. (1978). 176 с.

[26] M.S. Kennedy, N.R. Moody, D.P. Adams, M. Clift, D.F. Bahr. Mater. Sci. Eng. A 493, 299 (2008).

[27] Ю.В. Найдич, И.И. Габ, Б.Д. Костюк, Т.В. Стецюк, Д.И. Куркова, С.В. Дукаров. Докл. НАН Украины 5, 97 (2007).

[28] D. Holec, P.H. Mayrhofer. Scr. Mater. 67, 760 (2012).

[29] A. Navrotsky. Geochem. Trans. 4, 34 (2003).

[30] C.M. Balik, S.K. Tripathy, A.J. Hopfinger. J. Polym. Sci. B 20, 2003 (1982).

[31] G. Guisbiers, D. Liu, Q. Jiang, L. Buchaillot. Phys. Chem. Chem. Phys. 12, 7203 (2010).

[32] A.A. Stekolnikov, J. Furthmüller, F. Bechstedt. Phys. Rev. B 65, 115318 (2002).

[33] B.W. Veal, A.P. Paulikas. Phys. Rev. B 10, 1280 (1974).

[34] I.G. Gurtubay, J.M. Pitarke, Wei Ku, A.G. Eguiluz, B.C. Larson, J. Tischler, P. Zschack, K.D. Finkelstein. Phys. Rev. B 72, 125117 (2005).

[35] N.F. Mott. Proc. R. Soc. A 153, 699 (1936).

[36] N. Oka, R. Arisawa, A. Miyamura, Y. Sato, T. Yagi, N. Taketoshi, T. Baba, Y. Shigesato. Thin Solid Films 518, 3119 (2010).

[37] F. Nitsche, B. Schumann. J. Low. Temp. Phys. 39, 119 (1980).

[38] В.А. Красильников, В.В. Крылов. Введение в физическую акустику. Наука, М. (1984). 400 с.

[39] O.M. Rayleigh. Proc. R. Soc. A 79, 399 (1907).

[40] В.Н. Беломестных. Письма в ЖТФ 30, 14 (2004).

[41] K. Gordiz, A. Henry. Sci. Rep. 6, 23139 (2016). 